\section{Improving Cerebral Cortical Magnetic Resonance Imaging Using a Readily Available Surface Coil}

\begin{abstract}
A B S T R A C T
Subtle structural deformities of the cerebral cortex have been shown to be the cause of seizures in patients with refractory epilepsy. Brain imaging using high-resolution focused protocols with standard head coils may not provide sufficient image quality needed for evaluating subtle cortical abnormalities. The authors describe the use of a readily available shoulder coil placed over a specific area of the brain that has been clinically determined to enhance the signal to noise and resolution of the cortical surface. Delineating the cortical surface using a shoulder coil can help to detect subtle areas of cortical thickening, blurring of the gray-white matter junction, or focally abnormal gyral and sulcal patterns.
\end{abstract}

Key words: Epilepsy, surface coil MRI, cortical dysplasia.

Gomez-Hassan DM, Blaesing C, Chenevert TL, Shattock S. Improving cerebral cortical magnetic resoning imaging using a readily available surface coil. J Neuroimaging 2004;14:231-234. DOI: $10.1177 / 1051228404264955$

Identifying structural abnormalities of the brain's cortical surface is essential for patients who suffer from medically refractory neocortical epilepsy. Subtle cortical abnormalities may remain occult by imaging even with epilepsyfocused protocols using standard head coils. Recently, techniques have been developed to provide highresolution and significantly improved signal-to-noise

Received December 5, 2003, and in revised form February 4, 2004. Accepted for publication February 18, 2004.

From the Department of Radiology, University of Michigan Hospitals, Ann Arbor.

Address correspondence to Dr Diana M. Gomez-Hassan, Department of Radiology, University of Michigan Hospitals, 1500 East Medical Center Drive, Ann Arbor, MI 48109. E-mail: dmgh@umich.edu.
Diana M. Gomez-Hassan, MD, PhD

Cathy Blaesing, RT

Steven Shattock, RT

Thomas L. Chenevert, PhD imaging of detailed structures such as the orbits and optic nerves, the brachial plexus, and the carotid arteries using surface coil imaging. ${ }^{1-3}$ The brain's cortical surface also has been imaged using custom-designed phased array surface coils with enhanced resolution. ${ }^{4-7}$ In this report, we use a readily available shoulder coil to image the brain's cortical surface, with substantially improved image quality relative to head coil protocols.

\section{Technique}

Six patients with medically refractory epilepsy were initially evaluated by magnetic resonance imaging (MRI) using a G.E. Medical Systems 1.5 Tesla unit and an epilepsy-focused protocol that uses a standard birdcage design, transmit-receive head coil, and $256 \times 256$ matrix, with the associated parameters: repetition time/echo time/slice thickness/interspace. Imaging sequences for this protocol included coronal and axial fluid-attenuated inversion recovery $(10000 / 104 / 4.0 / 0.0)$ with an inversion time of $2200 \mathrm{msec}$, coronal T2 (7000/95/3.0/1.0), coronal T1 gadolinium-enhanced (500/16/5.0/1-2.0), and thinsection 3-dimensional coronal fast spoiled gradient (IR PREP) sequences (12.5/5.5/1.7/0.0) flip angle of $20^{\circ}$ and prep time of $300 \mathrm{msec}$. These select patients had undergone repeat imaging with the same scanner but used a shoulder array coil (MRI Devices, Waukesha, WI) placed and secured over a region of interest defined by previous encephalographic monitoring. A multiplane T1 localizer was initially performed followed by coronal, axial, and sagittal fast spoiled gradient-echo images using the same parameters as defined above except for a $256 \times 224$ matrix. In each of these cases, the signal-to-noise image quality of the cortical surface within the region of interest was significantly improved by the use of the shoulder coil (Fig 1). To date, only 2 patients in this group have had surgical resection of a cortical region suggested to be abnormal by surface coil imaging, and these areas proved to be abnormal pathologically (Fig 2). A qualitative evaluation of the region was performed to assess the degree of cortical thickening and blurring of the gray-white matter interface. 

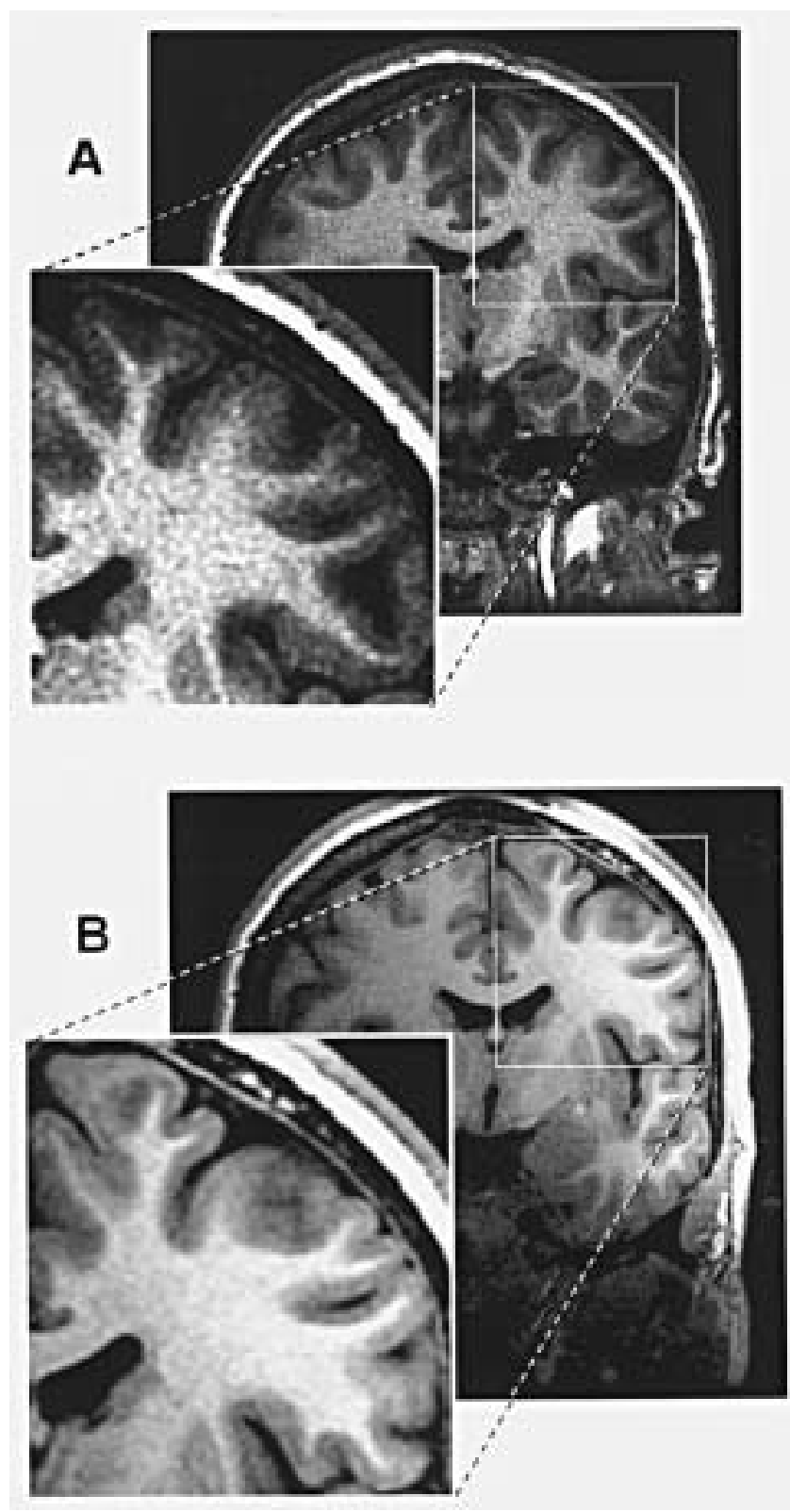

Fig 1. Coronal fast spoiled gradient inversion recovery sequence (TR 12.5, TE 5.5, 1.7 thk, 0sp) using a standard head coil provides uniform delineation of the gray-white interface in this normal brain (A). Repeat imaging using the same sequence and parameters but with a shoulder coil placed over the left frontal lobe provides greater image quality of the cortical surface and gray-white interface immediately beneath the coil (B).

\section{Discussion}

Malformations of cortical development have been shown to cause epilepsy. Identifying large malformations can usually be accomplished with standard and epilepsyfocused protocols using whole-brain head coils, but often, these techniques are limited in evaluating the cortical sur- face for subtle abnormalities. The goal of the neuroradiologist in the evaluation of these patients is to identify any potential structural abnormality especially in those areas defined by clinical and electrophysiologic monitoring. High-resolution imaging using dedicated epilepsy protocols are necessary to evaluate the entire brain for potential abnormalities. ${ }^{8}$ By finding abnormalities especially with regard to the mesial temporal structures that are concordant with the clinical data, potentially curable resections can be offered. Such imaging protocols are often sufficient for identifying pathology in the mesial temporal structures. However, even the high-resolution protocols may not reveal a lesion outside the mesial temporal structures. For those patients who show no lesion even with the high-resolution whole-brain protocols, surface coil imaging should be considered if sufficient localizing data such as electroencephalographic monitoring or functional imaging studies suggest a certain area of the brain. The improved signal to noise and resolution may be obtained with surface coil imaging, which allows better gray-white matter delineation than standard head coil imaging. ${ }^{3,6-7}$ Potential areas of thickening, abnormal gyral patterns, or blurring of the gray-white interface can be identified more readily with this improvement. Most facilities do not have a readily available coil laboratory in which to design specialized head surface coils. However, the shoulder coil, one that is available at most MRI centers, can offer added sensitivity as required for the epilepsy-focused exam.

Although this technique is beneficial to identifying cortical malformations immediately beneath the surface coil, there are certain limitations that need to be considered. Signal reduction occurs in areas deep and distal to the coil. Therefore, comparison to the contralateral side or evaluation for symmetry is more difficult using this coil. In addition, the temporal lobes are more difficult to evaluate because the contour of the coil is not ideally suited for placement on the side of the head. A customized surface coil, rather than a shoulder coil, may be better suited to evaluate temporal lobe abnormalities if the standard epilepsy focus protocols are insufficient. ${ }^{5}$ But in all other areas of the cortical surface, this coil can be readily secured on the patient's head. Despite the more limited coverage of the brain's surface compared to routine head coils, the shoulder coil offers a wider coverage of the cortex compared to the small 3- or 5-in coils used for the temporomandibular joint. In our experience, identifying subtle abnormalities is often aided by direct comparison with normal-appearing cortex immediately adjacent to an area of distortion. The very small coils may be too limited in focus and may optimize imaging over only 1 or 2 gyri, thereby preventing detection of the changes in the cortex. 

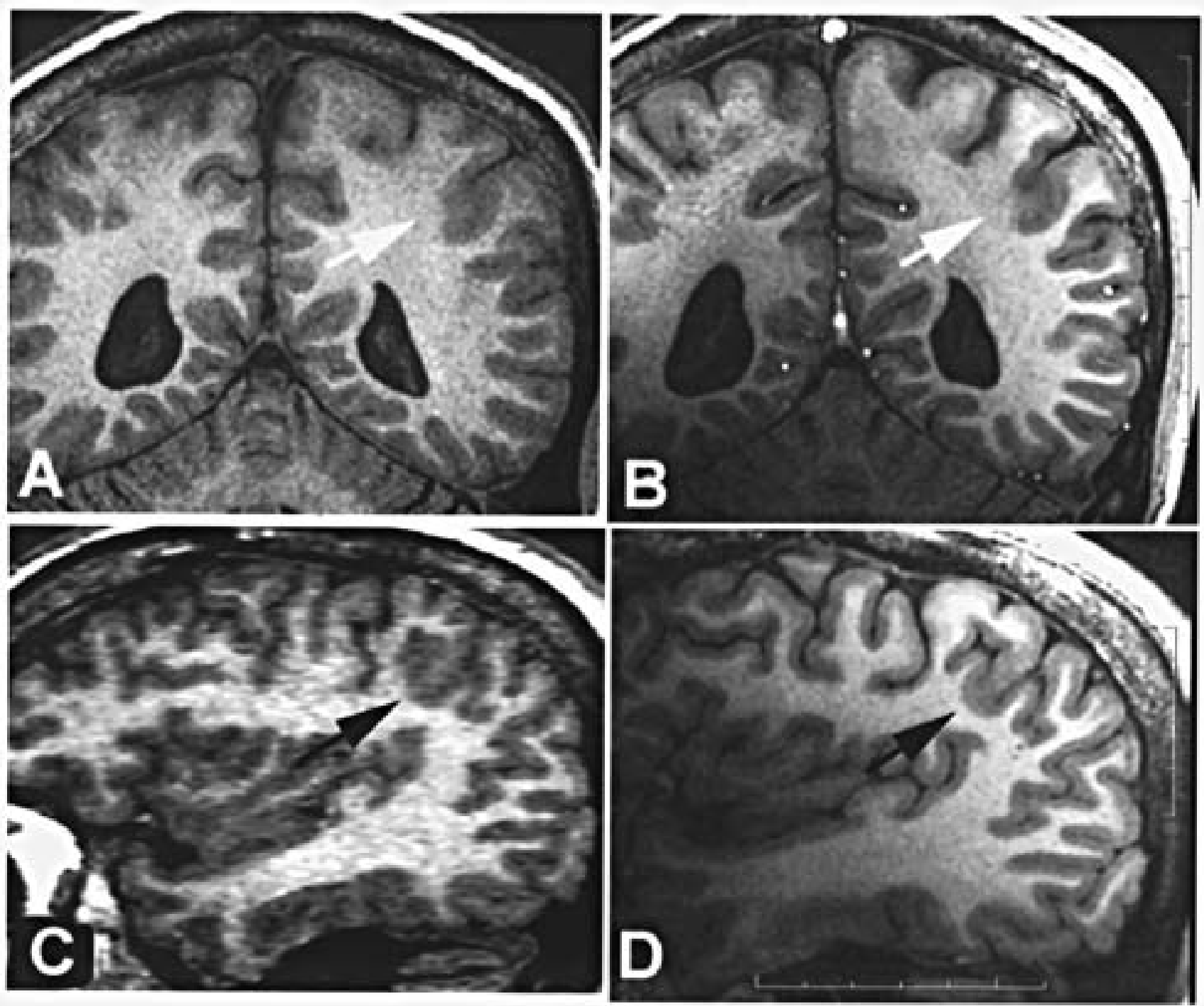

Fig 2. Coronal $(A)$ and reformatted sagittal $(C)$ fast spoiled gradient inversion recovery sequence obtained using a standard head coil is suboptimal in identifying an area of cortical thickening along the left middle parietal sulcus, whereas coronal (B) and sagittal (D) surface coil imaging better delineates an area of cortical thickening. This region was identified as the epileptic focus with invasive monitoring and was shown to be pathologically consistent with cortical dysplasia after surgical resection.

Last, a negative surface coil imaging study does not exclude the presence of an epileptogenic focus. Rather, the focus may be occult by MRI or located elsewhere in the brain, but even this negative information is important in guiding clinical management of these patients. By no means should the surface coil be used as a screening tool to identify a lesion. Rather, it can be used to provide supportive data that would help guide clinical and possibly surgical management. ${ }^{9}$

The sensitivity and specificity of the surface coil awaits further analysis with other patients and their postsurgical follow-up. By offering patients for whom initial evaluation with high-resolution epilepsy-focused MRIs were negative with this specialized and focused technique, a greater number of potential lesions can be identified. This may ultimately save a patient from undergoing increased morbidity and mortality associated with invasive electroencephalographic monitoring and even offer patients the possibility of a seizure-free outcome if potentially resectable lesions are identified. For the practicing community and for those institutions that are not equipped with specialized surface coils, the shoulder coil can be easily used for a more focused evaluation of the cerebral cortex. 


\section{References}

1. Breslau J, Dalley RW, Tsuruda JS, Hayes CE, Maravilla KR. Phased-array surface coil MR of the orbits and optic nerves. AJNR Am J Neuroradiol 1995;16(6):1247-1251.

2. Hayes CE, Tsuruda JS, Mathis CM, Maravilla KR, Kliot M, Filler AG. Brachial plexus: MR imaging with a dedicated phased array of surface coils. Radiology 1997;203(1):286289.

3. Hayes CE, Mathis CM, Yuan C. Surface coil phased arrays for high-resolution imaging of the carotid arteries. J Magn Reson Imaging 1996;6(1):109-112.

4. Hayes CE, Tsuruda JS, Mathis CM. Temporal lobes: surface MR coil phased-array imaging. Radiology 1993;189(3):918920.
5. Grant PE, Barkovich AJ, Wald LL, Dillon WP, Laxer KD, Vigneron DB. High-resolution surface-coil MR of cortical lesions in medically refractory epilepsy: a prospective study. AJNR Am J Neuroradiol 1997;18:291-301.

6. Wald LL, Carvajal L, Moyher SE, Nelson SJ, et al. Phased array detectors and an automated intensity-correction algorithm for high-resolution MR imaging of the human brain. Magn Reson Med 1995;34(3):433-439.

7. Moyher SE, Vignerson DB, Nelson SJ. Surface coil MR of the human brain with an analytic reception profile correction J Magn Reson Imaging 1995;5(2):139-144.

8. Weishman UC. Clinical applications of neuroimaging in epilepsy. J Neurol Neurosurg Psychiatr 2003;74:466-470.

9. Edwards JC, Wyllie E, Ruggeri PM, et al. Seizure outcome after surgery for epilepsy due to malformation of cortical development. Neurology 2000;55(8):1110-1114. 